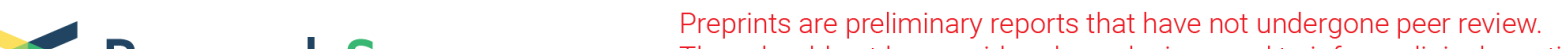 Research Square
or referenced bot be considered conclusive, used to inform clinical practice,
os validated information.
}

\section{Longitudinal evaluation of five nasopharyngeal carcinoma animal models on the microPET/MR platform}

\section{Jingjing Shi}

University of Hong Kong Li Ka Shing Faculty of Medicine

\section{Zhichao Xue}

National Institute of Metrology

Kel-Vin Tan

University of Hong Kong Li Ka Shing Faculty of Medicine

Hui Yuan

Guangdong Provincial People's Hospital

Anna Chi Man Tsang

The Chinese University of Hong Kong

\section{Sai Wah Tsao}

University of Hong Kong Li Ka Shing Faculty of Medicine

Pek-Lan Khong ( $\nabla$ dnrkpl@nus.edu.sg)

National University of Singapore

\section{Research Article}

Keywords: Nasopharyngeal carcinoma, Patient-derived xenografts, microPET/MR, Tumour growth pattern, [18F]FDG, Necrosis

Posted Date: October 5th, 2021

DOI: https://doi.org/10.21203/rs.3.rs-934896/v1

License: (c) (1) This work is licensed under a Creative Commons Attribution 4.0 International License.

Read Full License

Version of Record: A version of this preprint was published at European Journal of Nuclear Medicine and Molecular Imaging on December 4th, 2021. See the published version at https://doi.org/10.1007/s00259021-05633-4. 


\section{Abstract \\ Purpose}

We longitudinally evaluated the tumour growth and metabolic activity of two well-established nasopharyngeal carcinoma (NPC) animal models (C666-1, C17) and three novel models (Xeno76, Xeno23 and NPC43) using a microPET/MR system. With a better understanding of the interplay between tumour growth and metabolic characteristics of these NPC models, we aim to provide insights for the selection of appropriate NPC cell line/xenograft models to assist novel drug discovery and evaluation.

\section{Methods}

Mice were imaged by $\left[{ }^{18} \mathrm{~F}\right] \mathrm{FDG}$ microPET/MR twice a week for consecutive $3-7$ weeks. $\left[{ }^{18} \mathrm{~F}\right] \mathrm{FDG}$ uptake was quantified by standardized uptake value (SUV) and presented as SUVmean tumour-to-liver ratio (SUVRmean). Longitudinal tumour growth patterns and metabolic patterns were recorded. SUVRmean and histological characteristics were compared across the five NPC models. Cisplatin was administrated to one selected optimal tumour model, $\mathrm{C} 17$ to evaluate our imaging platform.

\section{Results}

We found variable tumour growth and metabolic patterns across different NPC tumour types. C17 has an optimal growth rate and higher tumour metabolic activity compared with C666-1. C666-1 has a fast growth rate but is low in SUVRmean at endpoint due to necrosis as confirmed by H\&E. NPC43 and Xeno76 have relatively slow growth rates and are low in SUVRmean, due to severe necrosis. Xeno23 has the slowest growth rate, and a relative high SUVRmean. Cisplatin showed the expected therapeutic effect in the $\mathrm{C} 17$ model in marked reduction of tumour size and metabolism.

\section{Conclusion}

Our study establishes an imaging platform that characterizes the growth and metabolic patterns of different NPC models, and the platform is well able to demonstrate drug treatment outcome supporting its use in novel drug discovery and evaluation for NPC.

\section{Introduction}

Nasopharyngeal carcinoma (NPC) is one of the most prevalent malignant diseases among the population in East and Southeast Asia, particularly in the ethnic Cantonese population in southern China. In 2020, more than 133,000 new cases of NPC were diagnosed leading to approximately 80,000 deaths in the same year [1]. Whilst the prognosis of NPC has largely improved due to advancements in treatment in the past decades [2], more than $10 \%$ of patients still develop local recurrence and distant metastasis after 
primary treatment. Hence, management of advanced and recurrent disease, including the use of novel and targeted therapy remains an important topic in NPC $[3,4]$. Positron emission tomography (PET) imaging has been used widely in the clinics to evaluate metabolic activity in tumours, and it has been found useful in the evaluation of treatment outcome and in prognostication of NPC [5-7]. With the advent of positron emission tomography and magnetic resonance (PET/MR), it is now recommended that NPC patients undergo a one-stop evaluation for both metabolic and anatomical information using PET/MR [810]. In order to enable rapid translation of novel cancer drugs from discovery to clinical trial evaluation, the potential of microPET/MR in small animal imaging is of interest for early in vivo testing of these drugs and for performing co-clinical trials [11-13].

To better understand the underlying pathogenesis of the disease as well as to develop novel treatment strategies, patient-derived xenografts (PDXs) have been used as important models in pre-clinical studies. In NPC research, XenoC15 and XenoC17 represent the most widely used PDXs [14] and the other two available PDXs are Xeno2117 and Xeno666 [15]. These have been passaged for over 25 years and are expected to have already lost their original genetic and pathological properties. Among the conventional NPC cell lines that have been available in the past decade, only C666-1 is Epstein-Barr virus (EBV) positive [16] and is hence, able to be more representative of clinical NPC. In recent years, Tsao's group has found success in establishing several new NPC xenografts and cell lines for investigation; cell line $\mathrm{C} 17$ was derived from xenograft of metastatic NPC specimen [17]; Xeno76 was derived from primary NPC specimen (7); Xeno23 and cell line NPC43 were both derived from recurrent NPC specimens [18]. Although the genetic features of these new NPC PDXs have been reported [18], the longitudinal growth pattern and the metabolic characteristics still remain unknown. Considering the ability of xenografts retaining histopathological and molecular features from the parental tumours [19] and tumour metabolic heterogeneity that has been reported across NPC tumours [20,21], it is reasonable to assume that PDXs from different patients may have diverse metabolic patterns.

In this study we characterize and compare the tumour growth and metabolic activity in a time dependent manner, of two well-established NPC models (C666-1, C17) and three novel models (Xeno76, Xeno23 and NPC43) using a microPET/MR system. Based on our findings, we subsequently select the most appropriate model and through the use of a well-established drug, we assess the utility of the imaging platform in the longitudinal evaluation of drug response in the tumour model. Our overarching goal is to establish an imaging platform that describes the growth and metabolic characteristics of NPC models, establish tumour imaging metrics and provide insights for the selection of appropriate NPC cell line/xenograft models to assist novel drug discovery and evaluation.

\section{Materials And Methods}

\section{Cancer cell line models and xenografts}

For cancer cell lines that derived from xenografts, cells were cultured and expanded in Roswell Park Memorial Institute (RPMI) with 10\% fetal bovine serum (FBS) and 1\% penicillin and streptomycin. C666-1 
cell line was obtained from Professor Dolly Huang (Chinese University of Hong Kong). C17 and NPC43 cell lines were newly established in Tsao's lab $[17,18] .10^{7}$ cells were resuspended in $200 \mu$ l Matrigel in 1:1 (vol:vol) ratio, and the mixture were subcutaneously injected into the right loin of each NOD.CB17Prkdcscid/J mouse (male, 4-5 weeks old, $\mathrm{n}=5$ for each model). For xenograft derived from tumour blocks, tumour from donor mouse was minced into fragments of $2 \mathrm{~mm}$ in diameter. Then wet fragments were subcutaneously implanted in the right loin of recipient mice (male, 4-5 weeks old, $n=5$ for each model). All animal experiments were conducted according to the animal license issued by Hong Kong Department of Health and with the approval of the Committee on the Use of Live Animals in Teaching and Research (CULATR) of The University of Hong Kong.

\section{Drug treatment}

Cisplatin (Selleckchem, S1166) was diluted with dimethylformamide (DMF) to a stock concentration of $20 \mathrm{mM}$ and stored in $4{ }^{\circ} \mathrm{C}$ avoiding light. Tumour mice were randomized into drug treatment group or vehicle group when tumours reach $50-100 \mathrm{~mm}^{3}$. Then, the stock solution of Cisplatin was further diluted with saline into a concentration of $4 \mathrm{mg} / \mathrm{kg}$ and was given to animals weekly for consecutive 3 weeks by intraperitoneal injection. Tumour volume was measured three times a week using calipers throughout the entire duration of treatment. Animals were sacrificed after the treatment period and tumours were harvested for further histopathological study and immunohistochemistry.

\section{$\left[{ }^{18}\right.$ F $]$ FDG microPET/MR scan}

After cancer cells injection or tumour fragment implantation, microPET/MR monitoring commenced when the tumour was palpable on each mouse. Mice were fasted overnight with free access to water before microPET/MR scans. Mice were anaesthetized using a mixture of medical air and isoflurane (induction $5 \% \mathrm{v} / \mathrm{v}$; maintenance $2.0-2.5 \% \mathrm{v} / \mathrm{v}$ ) and placed on the pre-heated nanoScan ${ }^{\circledR} 3 \mathrm{~T}$ PET/MR scanner (Mediso Medical Imaging Systems Ltd., Budapest). $9.25 \pm 0.37 \mathrm{MBq}{ }^{18} \mathrm{~F}$-deoxyglucose ([ $\left.\left.{ }^{18} \mathrm{~F}\right] \mathrm{FDG}\right)$ was injected via lateral tail vein. Animals were scanned twice a week for consecutive 3-7 weeks till the humane endpoint using the same protocol (Fig. S1). T1 and T2 weighted imaging (T1WI and T2WI) were performed on all the mice for tumour size assessment (Table S1). A 20-minute static PET scan was performed 60 minutes after injection of radiotracer (Table S2). For drug treatment assessment, $\left[{ }^{18} \mathrm{~F}\right] \mathrm{FDG}$ PET scan was performed pre- and post-treatment for tumour uptake comparison.

\section{Autoradiograph}

Tumour was exposed from the mouse body and marked using tissue dye to match the orientation of $\mathrm{PET} / \mathrm{MR}$ images. Tumours were then extracted and cut into $3 \mathrm{~mm}$ thick sections. Parts of the tumour were quickly embedded in Tissue-Tek medium (Sakura Co., Ltd., Tokyo, Japan) and snap-freezed in the liquid nitrogen. Frozen tumours were sliced into $10 \mu \mathrm{m}$ sections using cryostat (CM1950 Leica Biosystems, German) and covered with plastic wrap. Tumour slices were placed in a cassette together with a phosphor screen and one hour exposure was performed to detect the distribution of $\left[{ }^{18} \mathrm{~F}\right] \mathrm{FDG}$. 
Then the phosphor screen was scanned using Typhoon5 Biomolecular Imager (GE Amersham, UK) with a resolution of $25 \mu \mathrm{m}$. Image $\mathrm{J}$ was used to further process the images.

\section{Histological studies}

Each tumour section was cut into $5 \mu \mathrm{m}$ thick slices and baked in $37^{\circ} \mathrm{C}$ oven overnight for hematoxylin and eosin (H\&E) staining and immunohistochemical staining. Ki-67 (1:100; Santa Cruz, sc-23, 900) was stained as a cell proliferation marker. To prepare for immunohistochemistry, the antigen was retrieved by immersing tumour slices into the boiling sodium citrate buffer $(10 \mathrm{mM}, \mathrm{pH} 6.0)$ for 20 minutes. Tumour slices were incubated with $3 \%$ bovine serum albumin at room temperature for 10 minutes and incubated with primary antibody in a wet box overnight. On the second day, the slices were incubated with $3 \% \mathrm{H}_{2} \mathrm{O}_{2}$ for 8 minutes and secondary antibody was applied to the slices for 1 hour. DAB (Dako, Cat. \#: K346711-2) substrate was applied to the slices till brown color developed. The slices were dehydrated and mounted with Permount (Dako, Cat. \#: S3023). All the slices were scanned with color imaging microscope (Olympus DP74). Image $\mathrm{J}$ was used to calculate the identified non-necrotic tumour region to the intact tumour region with a fixed threshold and QuPath software (University of Edinburgh, UK) [22] was used to detect the positive cells.

\section{Image analysis}

Static PET images were reconstructed on the Mediso Medical Imaging Systems using the build-in reconstruction algorithm Tera-Tomo 3D. PET images were anatomically coregistered with MRI images and fused images were viewed on Interview Fusion version 3.03.089.0000 (Mediso Medical Imaging Systems Ltd.). Volume of interests (VOIs) of liver and tumour were manually drawn on the images. $\left[{ }^{18} \mathrm{~F}\right] \mathrm{FDG}$ uptake was quantified by standardized uptake value (SUV) as described in the previous publication [23]. For comparable analysis, the hepatic [ $\left.{ }^{18} \mathrm{~F}\right] \mathrm{FDG}$ uptake was used as an internal reference background for VOI quantification [24]. The tumour SUVmax and SUVmean were normalized by SUVmean_liver and presented as SUVmax_ratio (SUVRmax) and SUVmean_ratio (SUVRmean) using the equation: [25].

\section{Statistical analysis}

Statistical analysis and figures were performed and drawn using Prism (GraphPad, Inc.). Tumour growth rate was calculated by fitting tumour volume at each time point into the exponential growth equation [26], where is the tumour volume at time, is the tumour volume at initial timepoint, and is the tumour growth rate. Tumour doubling time was calculated using the exponential growth equation. Data are presented as mean values \pm standard errors of the means. Relations between SUV and tumour growth rate, as well as histological staining and SUV were analyzed using Pearson's correlation. Comparison between pre-treatment and post-treatment was done by two-tailed Student's $t$-test. A $p$-value lower than 0.05 was considered statistically significant.

\section{Results}




\section{Growth patterns of NPC animal models}

We found that tumours from cell line models: C17, C666-1 and NPC43 started to grow as early as week2 post implantation, while tumours from fragment implantation, Xeno76 and Xeno23, needed a relative longer time to initiate growth and to reach humane endpoint. To compare among models, a 4-week observation period was selected for each model when tumours reached a comparable size ( $100-200 \mathrm{~mm}^{3}$ ): week2 to week5 for C17/C666-1/NPC43/Xeno76; and week11 and week14 for slow growing model Xeno23. Within the observation period, all five models attained varying tumour volumes (Fig. 1a and b). Notably, tumours from NPC cell lines (C17 and C666-1) generally were found to have a faster growth rate and shorter doubling time compared with tumours grown from tumour fragment implantation (Xeno76 and Xeno23). C666-1 had the fastest tumour growth rate of $12.7 \pm 0.4$ (\%/day) and tumour doubling time of $5.5 \pm 0.2$ days (Table 1), resulting in tumour oversize at a relative early time point. Xeno76 had a tumour growth rate of $7.3 \pm 0.8$ (\%/day) and tumour doubling time of 10.1 \pm 1.5 days. Xeno23 was the slowest in tumour growth, with a rate of $2.9 \pm 0.6(\% /$ day) and tumour doubling time of $27.7 \pm 4.8$ days. After 3-4 weeks, the individual difference of tumour volume were relatively large within C17 and C666-1: ranging from $500-1100 \mathrm{~mm}^{3}$ for $\mathrm{C} 17$ and $600-900 \mathrm{~mm}^{3}$ for C6661. However, this did not lead to a big standard deviation in tumour growth rate when fitting into the exponential growth curve, suggesting that the growth pattern is relative stable and distinct for each xenograft model.

\section{Repeatability of $\left[{ }^{18} \mathrm{~F}\right] \mathrm{FDG}$ microPET/MR imaging system for SUV measurement}

To investigate the metabolic activity of NPC xenograft models, we first tested the repeatability of the microPET/MR system. The glucose uptake of liver in mice was reported stable upon fasting condition in several studies $[27,28]$ and SUVmean_liver has been used as the background to normalize radiotracer accumulation [29]. Thus, in our study SUVmean_liver was used to examine the repeatability of the microPET/MR imaging system. We analyzed the initial liver uptake and endpoint liver uptake for all five mice models and a total of 50 data points were included in the analysis. Bland-Altman plot (Fig. 2) showed mean difference between the two timepoints was 0.0196 (95\% limit of agreement: -0.099 - 0.14 ). Coefficient of variation (CoV) for SUVmean_liver between the initial and endpoint was $6.95 \% \pm 5.02 \%$. All the data points were within the agreement interval. No significant differences were observed between the initial and endpoint liver uptake. Results show an excellent consistency of liver SUVmean, suggesting the microPET/MR imaging system is stable and able to produce repeatable results.

\section{Metabolic patterns of NPC animal models}

We monitored the longitudinal changes in tumour uptake and found highly variable tumour metabolic patterns across tumour types. SUVRmean and SUVRmax of C17 showed a continuous increasing pattern, while C666-1 showed a decreasing pattern (Fig. 3a) attributed to extensive necrosis confirmed by histology at the endpoint. For Xeno76, NPC43 and Xeno23, there were no obvious overall changes in tumour metabolism over time. To further investigate whether tumour metabolism may change in a later 
timepoint, we continued to monitor tumour uptake of Xeno76 till week8 when the mice reached humane endpoint, still we did not observe any significant changes in the tumour uptake. After 4-week observation, $\mathrm{C} 17$ has the highest SUVRmean $(3.19 \pm 0.92)$ and Xeno76 is the lowest $(1.57 \pm 0.28)$. There was a significant difference between $\mathrm{C} 17$ and Xeno76 in SUVRmean $(p=0.0476)$, suggesting the metabolic heterogeneity across NPC xenograft models. We also compared the change of SUVRmean over the 4week period (Fig. $3 b$ ) and found good correlation between growth rate and absolute change in SUVRmean (Fig. $3 c, r=0.6340, p=0.0009$ ), regardless of tumour types and metabolic patterns. As expected, there was a larger standard deviation in SUVRmax than SUVRmean values due to the inherent characteristic of SUVmax compared to SUVmean measurements (Fig. 3b and d).

\section{Relation between PET, H\&E and Ki-67}

To compare PET images and autoradiography with H\&E staining, tumour was extracted at the endpoint and tumour slices went through histological analysis (Fig. 4a, b and c). The low uptake region shown on the PET images and autoradiography image was confirmed to be necrotic region by H\&E staining (Fig. 4d). We then performed a correlation between tumour SUV and the area of the non-necrotic region represented by H\&E staining, across all tumour types. Strong positive correlation was found between SUVRmean and the area of the non-necrotic region (Fig. 4e). Notably, this result revealed that the presence of necrotic regions is a cause of reduced overall $\left[{ }^{18} \mathrm{~F}\right] \mathrm{FDG}$ uptake in the tumour.

In addition, we analyzed the expression level of cell proliferation marker Ki-67 at the endpoint. Proliferating cells mainly accumulated in the outer layer of the tumours, which was consistent with the distribution of higher glycolytic regions on the PET images (Fig. 4a and c). Diverse expression levels of Ki67 were found among xenograft models. The expression level of Ki-67 was the highest in $\mathrm{C} 17$ and the lowest in C666-1 (Fig. 4f).

In summary, we found that $\mathrm{C} 17$ has an optimal growth rate, high SUVRmean, and this result was consistent with histology, which is high Ki-67 level and little necrosis confirmed by H\&E. C666-1 has a fast growth rate, relatively low Ki-67 level, low SUVRmean and much necrosis confirmed by H\&E. NPC43 and Xeno76 have slow growth rates and also low Ki-67 level and SUVRmean, as well as extensive necrosis confirmed by H\&E. Xeno23 has the slowest growth rate among these models, but it has a relative high Ki67 level and high SUVRmean. Notably, C17 has a much higher endpoint SUVRmean compared to the other models which had similar SUVRmean (Fig. 4g).

\section{Treatment assessment}

As a proof of concept, we applied standard chemotherapy for NPC, cisplatin, and evaluated tumour growth rate, metabolism and cell proliferation marker Ki- 67 before and after the treatment. Based on our findings of the NPC xenograft models, we selected C17 as the optimal tumour model for our purpose due to the satisfactory growth rate and high tumour metabolic activity which we observed over the 4-week period. $4 \mathrm{mg} / \mathrm{kg}$ cisplatin was administered for consecutive 4 weeks. The result of cisplatin treatment is shown in Fig. 5. During the treatment period, we observed a siginificant tumour inhibition effect by 
cisplatin. Statistical analysis showed significant differences in tumour volume (Fig. 5a and b) and SUVmean (Fig. 5c) between treatment group and vehicle group. Immunohistochemistry staining also confirmed the results. Our results showed a remarkably lower expression level of Ki-67 in cisplatin treated group, suggesting that tumour cells proliferation have been suppressed by cisplatin (Fig. 5d, e and f). Imaging using microPET/MR was able to demonstrate the metabolic and anatomical outcome of cisplatin treatment on the NPC model.

\section{Discussion}

A successful pre-clinical study would largely depend on the selection and use of suitable animal models to predict clinical efficacy [30-32]. As new EBV positive NPC xenograft models have been established recently $[17,18]$, more choices are available in selecting tumour models for experiments. The clear characterization of these tumour models would promote the appropriate model selection and therefore facilitate an improved outcome for pre-clinical studies. We aimed to depict and compare the growth pattern, the metabolic activity, and the histological characteristics of these models. Firstly, we found that xenografts derived from cancer cell lines (C666-1 and C17) tend to initiate tumour growth in an early timepoint and have a faster growth rate compared with xenografts derived from tumour blocks (Xeno76 and Xeno23) (Fig. 1). This finding is consistent with previous studies done by Tsao's group [33]. Similar trend was also reported in colorectal cancer [34] and breast cancer [35], and the reason could be: cancer cells which were injected into the mouse, could interact with the local environment in three-dimensions and have easy access to the nutrients and oxygen, thus resulting in fast tumour growth [36]. On the other hand, for the implanted tumour blocks, only cells in the outer layer have direct access to the nutrients and oxygen, which could be the reason for slower tumour growth. Furthermore, we also observed different tumour growth rates within the three cell line xenografts: the two well-established models C666-1 and C17 have a similar growth rate that is much faster than newly established NPC43 (Table1). One potential reason is that both C666-1 and C17 were established from xenografts that have been passaged for over 25 years $[14,16]$. During years' of passaging, highly aggressive cells may be retained and passaged from parental tumours to the next generation, thus causing C666-1 and C17 to exhibit more aggressive growth compared with NPC43. Nevertheless, both C666-1 and C17 are EBV-positive (EBV +ve) in vitro and representative NPC models for investigations.

Pre-clinical imaging holds a key role in the non-invasive longitudinal evaluation of therapy response and in the establishment of novel drugs. Traditional drug studies in animal models use 'tumour size' as the standard to evaluate drug efficacy. Tumour size when applied as a single indicator has a lot of limitation, in most situations it is a surrogate marker for late therapy response compared to change of tumour metabolic activity. For instance, we found that C666-1 and C17 have a relative similar robust growth rates which could be measured through traditional method. However, using the microPET/MRI we first noted that with the similar growth rate, the two models could actually have a diverse metabolic activity (Fig. 3a, b and d). The decrease in tumour uptake in C666-1 can be explained by the early and extensive tumour necrosis which was observed by H\&E staining. In contrast, the uptake of C17 was in tandem with increase in tumour size and only relative small necrotic regions were observed at the endpoint. The distinct 
metabolic patterns may be related to the origin of the tumours: C666-1 was from a primary NPC [16] and C17 was from a cutaneous metastasis of a poorly differentiated NPC [17]. Previous studies suggested that cancer cells experience profound metabolic reprogramming due to the pressures exerted on cells from the microenvironment during metastasis, thus leading to metabolic change and even genomic change from the primary tumour [37]. We found that SUVRmean was highest in the C17 model indicating a higher tumour metabolism, and low in the Xeno76 model, indicating a lower tumour metabolism. These findings were confirmed by cell proliferation marker Ki-67 in the specimen at sacrifice (Fig. 4f),indicating the major advantage of PET imaging in imaging metabolism of NPC xenograft models. In general, tumour metabolic activity plus tumour size would more comprehensively reflect the status of xenografts.

Xenograft models are essential for development of novel targeted therapy in NPC, which has been slow in development partially due to the lack of models, and suitable models that are able to retain their EBV episomes during passaging [18]. A robust xenograft model for longitudinal studies should not only have a suitable growth rate, but also maintain an appropriate metabolic activity, ie without overt necrosis. Based on our findings, C17 and C666-1 have an optimal tumour growth rate. The advantage of C17 over C666-1 is that $\mathrm{C} 17$ can maintain sufficient metabolic activity for longitudinal evaluation (Fig. 4g). For C666-1, however, the treatment effect might be compromised by the high level of necrosis. In 2019, Xue et al. showed that when giving the same dose of palbociclib treatment, $\mathrm{C} 17$ presented better treatment outcome than C666-1. A prior study performed by Gressette et al. in 2014 using XenoC17, found that the tumour model was sensitive to the treatment of cisplatin. To evaluate our imaging platform, cisplatin was administrated to $\mathrm{C} 17$ cell line xenograft and we found the platform useful to demonstrate the expected therapeutic results of cisplatin. Hence, we suggest $\mathrm{C} 17$ could be a robust xenograft model for treatment studies. As for C666-1, due to its defective in lytic EBV reactivation [38], it can represent a unique group of NPC patients and be used to develop treatment strategies targeting the induction of lytic reactivation. Further, because both cell lines and xenografts are available for C666-1, C17 and NPC43, they can be used to conduct matched in vitro/in vivo study to test the efficacy of novel drug treatment or targeted therapy [39]. For Xeno76, Xeno23 and NPC43, all these models have a relative slower growth rate and stable tumour metabolic activity throughout growth. When comparing Xeno76 and Xeno23, though both models take a long time to initiate tumour growth, Xeno76 grows quickly once the tumour reaches to palpable size and results in large necrotic regions, while Xeno23 remains small for over three months with relative less necrotic regions. For NPC43, though it has steady growth and metabolic activity, it was found to easily form necrotic regions and extensive cystic change were noted in several samples (Fig. S2). In case of need for slower growing tumour models, NPC43 and Xeno23 maybe considered.

This study demonstrated the distinct growth and metabolic patterns across different NPC xenografts, as well as illustrating the application of tumour imaging metrics using a microPET/MR system. Further investigations are needed to explore the molecular mechanism that resulted in the distinct characteristics of these tumour models. Moreover, the metabolic characteristics of the specific PDX model have the potential to guide its donor patient's treatment [40], and thus lead to a new era of personalized medicine. 


\section{Declarations}

\section{Funding information}

The research was supported by the University of Hong Kong Seed Fund for Basic Research (201910159081) and the Hong Kong Research Grants Council (RGC) Collaborative Research Grant (C7018-14E).

\section{Conflict of interest}

The authors have no relevant financial or non-financial interests to disclose.

\section{Availability of data and material}

The datasets used and/or analysed during the current study are available from the corresponding author on reasonable request.

\section{Code availability}

Not applicable

\section{Authors' contributions}

JS and ZX contributed to the study conception and design. JS conducted the microPET/MR experiments, image analysis and data analysis. ZX conducted the animal model estabolishment and histological study. The first draft of the manuscript was written by JS and all authors commented on previous versions of the manuscript. All authors read and approved the final manuscript.

\section{Ethics approval}

All animal experiments were conducted under conditions compliant with the animal license issued by the Hong Kong Department of Health and with the approval of the Committee on the Use of Live Animals in Teaching and Research (CULATR) of the University of Hong Kong (CULATR No. 4898).

\section{Consent to participate}

Not applicable

\section{Consent for publication}

All authors involved in the study provided their consent to the submission of this article for publication.

\section{Clinical Trial Registration}

Not applicable 


\section{References}

1. Sung H, Ferlay J, Siegel RL, Laversanne M, Soerjomataram I, Jemal A, et al. Global Cancer Statistics 2020: GLOBOCAN Estimates of Incidence and Mortality Worldwide for 36 Cancers in 185 Countries. CA: A Cancer. Journal for Clinicians. 2021;71:209-49. doi:https://doi.org/10.3322/caac.21660.

2. Lee A, Chow JCH, Lee NY. Treatment Deescalation Strategies for Nasopharyngeal Cancer: A Review. JAMA Oncology. 2021;7:445-53. doi:10.1001/jamaoncol.2020.6154.

3. Lee VHF, Lam KO, Lee AWM. Chapter 10 - Standard of Care for Nasopharyngeal Carcinoma (20182020). In: Lee AWM, Lung ML, Ng WT, editors. Nasopharyngeal Carcinoma: Academic Press; 2019. pp. 205-38.

4. Chan JYW, Lam TC, Ng WT. Chapter 13 - Salvage of Local Recurrence. In: Lee AWM, Lung ML, Ng WT, editors. Nasopharyngeal Carcinoma: Academic Press; 2019. pp. 289-312.

5. Huang B, Wong C-YO, Lai V, Kwong DL-W, Khong P-L. Prognostic Value of $<$ sup $>18</$ sup $>$ F-FDG PET-CT in Nasopharyngeal Carcinoma: Is Dynamic Scanning Helpful? BioMed Research International. 2015;2015:582614. doi:10.1155/2015/582614.

6. Alessi A, Lorenzoni A, Cavallo A, Padovano B, lacovelli NA, Bossi P, et al. Role of pretreatment 18FFDG PET/CT parameters in predicting outcome of non-endemic EBV DNA-related nasopharyngeal cancer (NPC) patients treated with IMRT and chemotherapy. La radiologia medica. 2019;124:41421. doi:10.1007/s11547-018-0980-6.

7. Chan S-C, Yeh C-H, Chang JT-C, Chang K-P, Wang J-H, Ng S-H. Combing MRI Perfusion and 18F-FDG PET/CT Metabolic Biomarkers Helps Predict Survival in Advanced Nasopharyngeal Carcinoma: A Prospective Multimodal Imaging Study. Cancers. 2021;13:1550.

8. Cheng Y, Bai L, Shang J, Tang Y, Ling X, Guo B, et al. Preliminary clinical results for PET/MR compared with PET/CT in patients with nasopharyngeal carcinoma. Oncol Rep. 2020;43:177-87. doi:10.3892/or.2019.7392.

9. Piao Y, Cao C, Xu Y, Huang S, Jiang F, Jin T, et al. Detection and staging of recurrent or metastatic nasopharyngeal carcinoma in the era of FDG PET/MR. European Archives of Oto-Rhino-Laryngology. 2021. doi:10.1007/s00405-021-06779-5.

10. Chan S-C, Yeh C-H, Yen T-C, Ng S-H, Chang JT-C, Lin C-Y, et al. Clinical utility of simultaneous wholebody 18F-FDG PET/MRI as a single-step imaging modality in the staging of primary nasopharyngeal carcinoma. Eur J Nucl Med Mol Imaging. 2018;45:1297-308. doi:10.1007/s00259-018-3986-3.

11. Chomet M, Schreurs M, Vos R, Verlaan M, Kooijman EJ, Poot AJ, et al. Performance of nanoScan $\mathrm{PET} / \mathrm{CT}$ and PET/MR for quantitative imaging of $18 \mathrm{~F}$ and $89 \mathrm{Zr}$ as compared with ex vivo biodistribution in tumor-bearing mice. EJNMMI Research. 2021;11:57. doi:10.1186/s13550-02100799-2.

12. Weerasekera A, Crabbé $M$, Tomé SO, Gsell W, Sima D, Casteels $C$, et al. Non-invasive characterization of amyotrophic lateral sclerosis in a hTDP-43A315T mouse model: A PET-MR study. Neurolmage: Clinical. 2020;27:102327. doi:https://doi.org/10.1016/j.nicl.2020.102327. 
13. Kim K, Kim H, Bae S-H, Lee S-Y, Kim Y-H, Na J, et al. [(18)F]CB251 PET/MR imaging probe targeting translocator protein (TSPO) independent of its Polymorphism in a Neuroinflammation Model. Theranostics. 2020;10:9315-31. doi:10.7150/thno.46875.

14. Busson P, Ganem G, Flores P, Mugneret F, Clausse B, Caillou B, et al. Establishment and characterization of three transplantable EBV-containing nasopharyngeal carcinomas. Int $\mathrm{J}$ Cancer. 1988;42:599-606. doi:https://doi.org/10.1002/ijc.2910420422.

15. Huang DP, Ho JHC, Chan WK, Lau WH, Lui M. Cytogenetics of undifferentiated nasopharyngeal carcinoma xenografts from southern chinese. Int J Cancer. 1989;43:936-9. doi:https://doi.org/10.1002/ijc.2910430535.

16. Cheung ST, Huang DP, Hui ABY, Lo KW, Ko CW, Tsang YS, et al. Nasopharyngeal carcinoma cell line (C666-1) consistently harbouring Epstein-Barr virus. International Journal of Cancer. 1999;83:121-6. doi:https://doi.org/10.1002/(SICI)1097-0215(19990924)83:1<121::AID-IJC21>3.0.C0;2-F.

17. Yip YL, Lin W, Deng W, Jia L, Lo KW, Busson P, et al. Establishment of a nasopharyngeal carcinoma cell line capable of undergoing lytic Epstein-Barr virus reactivation. Lab Invest. 2018;98:1093-104. doi:10.1038/s41374-018-0034-7.

18. Lin W, Yip YL, Jia L, Deng W, Zheng H, Dai W, et al. Establishment and characterization of new tumor xenografts and cancer cell lines from EBV-positive nasopharyngeal carcinoma. Nat Commun. 2018;9:4663. doi:10.1038/s41467-018-06889-5.

19. Prasetyanti PR, van Hooff SR, van Herwaarden T, de Vries N, Kalloe K, Rodermond H, et al. Capturing colorectal cancer inter-tumor heterogeneity in patient-derived xenograft (PDX) models. Int J Cancer. 2019;144:366-71. doi:https://doi.org/10.1002/ijc.31767.

20. Yang Z, Shi Q, Zhang Y, Pan H, Yao Z, Hu S, et al. Pretreatment 18 F-FDG uptake heterogeneity can predict survival in patients with locally advanced nasopharyngeal carcinoma--a retrospective study. Radiat Oncol. 2015;10:4. doi:10.1186/s13014-014-0268-5.

21. Chan S-C, Chang K-P, Fang Y-HD, Tsang N-M, Ng S-H, Hsu C-L, et al. Tumor heterogeneity measured on F-18 fluorodeoxyglucose positron emission tomography/computed tomography combined with plasma Epstein-Barr Virus load predicts prognosis in patients with primary nasopharyngeal carcinoma. Laryngoscope. 2017;127:E22-E8. doi:https://doi.org/10.1002/lary.26172.

22. Bankhead P, Loughrey MB, Fernández JA, Dombrowski Y, McArt DG, Dunne PD, et al. QuPath: Open source software for digital pathology image analysis. Sci Rep. 2017;7:16878. doi:10.1038/s41598017-17204-5.

23. Wong T-L, Ng K-Y, Tan KV, Chan L-H, Zhou L, Che N, et al. CRAF Methylation by PRMT6 Regulates Aerobic Glycolysis-Driven Hepatocarcinogenesis via ERK-Dependent PKM2 Nuclear Relocalization and Activation. Hepatology. 2020;71:1279-96. doi:https://doi.org/10.1002/hep.30923.

24. Wahl RL, Jacene H, Kasamon Y, Lodge MA. From RECIST to PERCIST: Evolving Considerations for PET Response Criteria in Solid Tumors. J Nucl Med. 2009;50:122S-50S. doi:10.2967/jnumed.108.057307. 
25. Shiono S, Abiko M, Okazaki T, Chiba M, Yabuki H, Sato T. Positron emission tomography for predicting recurrence in stage I lung adenocarcinoma: standardized uptake value corrected by mean liver standardized uptake value. Eur J Cardiothorac Surg. 2011;40:1165-9. doi:10.1016/j.ejcts.2011.02.041.

26. Lee JY, Kim M-S, Kim EH, Chung N, Jeong YK. Retrospective growth kinetics and radiosensitivity analysis of various human xenograft models. Laboratory animal research. 2016;32:187-93.

27. Hai W, Wu X, Shi S, Yang Y, Yang Z, Li B, et al. The effects of season change and fasting on Brown adipose tissue FDG-PET in mice. Biochem Biophys Res Commun. 2020;529:398-403.

28. Fueger BJ, Czernin J, Hildebrandt I, Tran C, Halpern BS, Stout D, et al. Impact of animal handling on the results of 18F-FDG PET studies in mice. J Nucl Med. 2006;47:999-1006.

29. Eschbach RS, Kazmierczak PM, Heimer MM, Todica A, Hirner-Eppeneder H, Schneider MJ, et al. 18 FFDG-PET/CT and diffusion-weighted MRI for monitoring a BRAF and CDK 4/6 inhibitor combination therapy in a murine model of human melanoma. Cancer Imaging. 2018;18:1-11.

30. Denayer T, Stohr T, Roy MV. Animal models in translational medicine: Validation and prediction. European Journal of Molecular Clinical Medicine. 2014;2:5-11.

31. Landi M, Everitt J, Berridge B. Bioethical. Reproducibility, and Translational Challenges of Animal Models. ILAR J. 2021. doi:10.1093/ilar/ilaa027.

32. Mergenthaler P, Meisel A. Chapter 8 - Animal models: value and translational potency. In: Wehling $M$, editor. Principles of Translational Science in Medicine (Third Edition). Boston: Academic Press; 2021. pp. 95-103.

33. Xue Z, Lui VWY, Li Y, Jia L, You C, Li X, et al. Therapeutic evaluation of palbociclib and its compatibility with other chemotherapies for primary and recurrent nasopharyngeal carcinoma. Journal of Experimental Clinical Cancer Research. 2020;39:1-24.

34. Julien S, Merino-Trigo A, Lacroix L, Pocard M, Goéré D, Mariani P, et al. Characterization of a Large Panel of Patient-Derived Tumor Xenografts Representing the Clinical Heterogeneity of Human Colorectal Cancer. Clin Cancer Res. 2012;18:5314-28. doi:10.1158/1078-0432.Ccr-12-0372.

35. Dunne LW, Huang Z, Meng W, Fan X, Zhang N, Zhang Q, et al. Human decellularized adipose tissue scaffold as a model for breast cancer cell growth and drug treatments. Biomaterials. 2014;35:49409.

36. Kapałczyńska M, Kolenda T, Przybyła W, Zajączkowska M, Teresiak A, Filas V, et al. 2D and 3D cell cultures-a comparison of different types of cancer cell cultures. Archives of medical science: AMS. 2018;14:910.

37. Lehuédé C, Dupuy F, Rabinovitch R, Jones RG, Siegel PM. Metabolic plasticity as a determinant of tumor growth and metastasis. Cancer research. 2016;76:5201-8.

38. Hau PM, Lung HL, Wu M, Tsang CM, Wong K-L, Mak NK, et al. Targeting Epstein-Barr virus in nasopharyngeal carcinoma. Front Oncol. 2020;10:600.

39. Pauli C, Hopkins BD, Prandi D, Shaw R, Fedrizzi T, Sboner A, et al. Personalized in vitro and in vivo cancer models to guide precision medicine. Cancer discovery. 2017;7:462-77. 
40. Cheng T, Zhan X. Pattern recognition for predictive, preventive, and personalized medicine in cancer. EPMA Journal. 2017;8:51-60.

\section{Tables}

Table 1. Tumour growth rate and in vivo doubling time.

\begin{tabular}{lll} 
Models & $\begin{array}{l}\text { Tumour Growth Rate } \\
\text { (\%/Day) }\end{array}$ & $\begin{array}{l}\text { Tumour Doubling Time } \\
\text { in vivo(Days) }\end{array}$ \\
\hline C17 & $11.9 \pm 0.5$ & $5.8 \pm 0.2$ \\
\hline C666-1 & $12.7 \pm 0.4$ & $5.5 \pm 0.2$ \\
\hline NPC43 & $6.0 \pm 0.8$ & $12.6 \pm 1.9$ \\
\hline Xeno76 & $7.3 \pm 0.8$ & $10.1 \pm 1.5$ \\
\hline Xeno23 & $2.9 \pm 0.6$ & $27.7 \pm 4.8$
\end{tabular}

Figures 


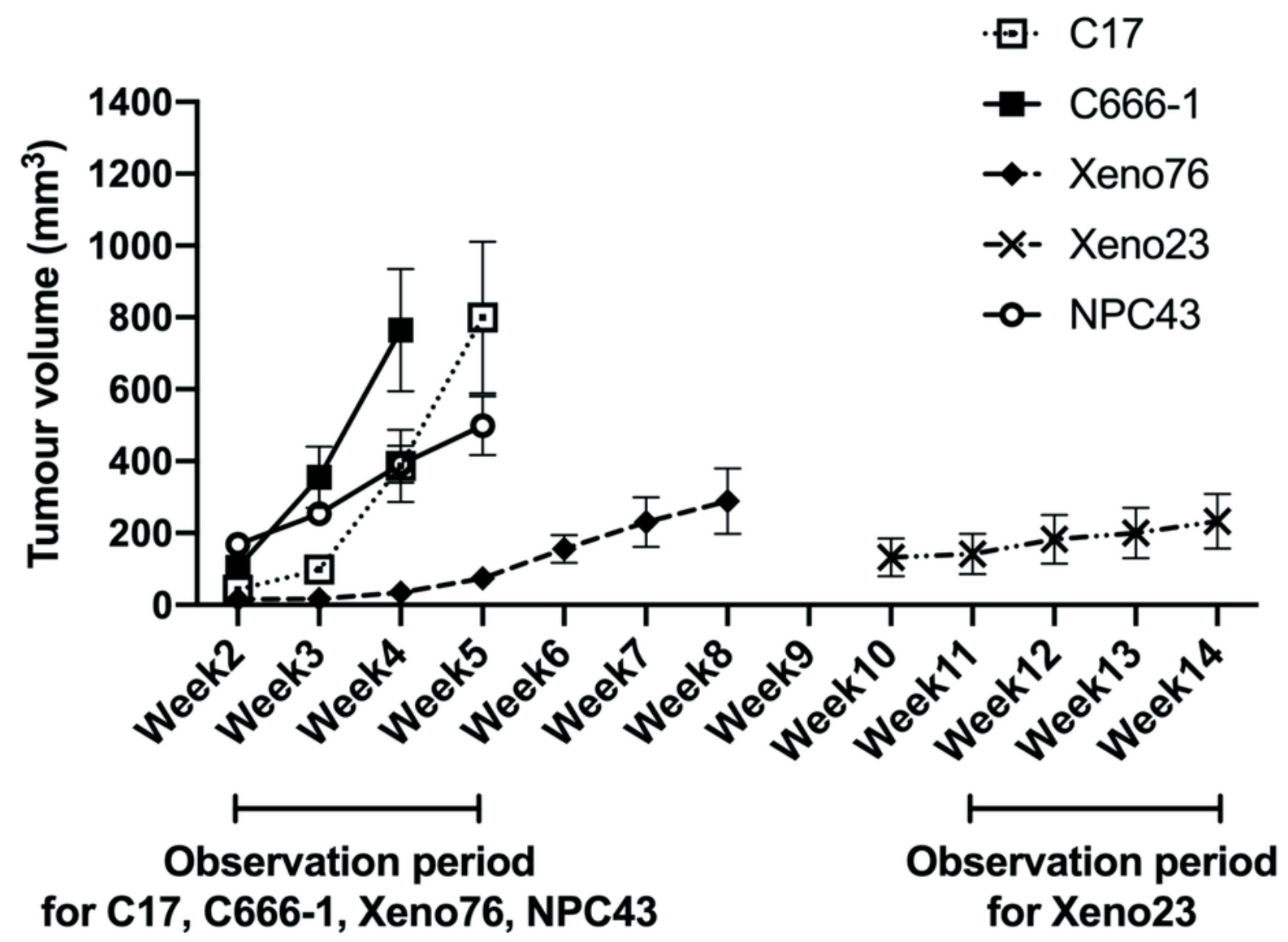

b

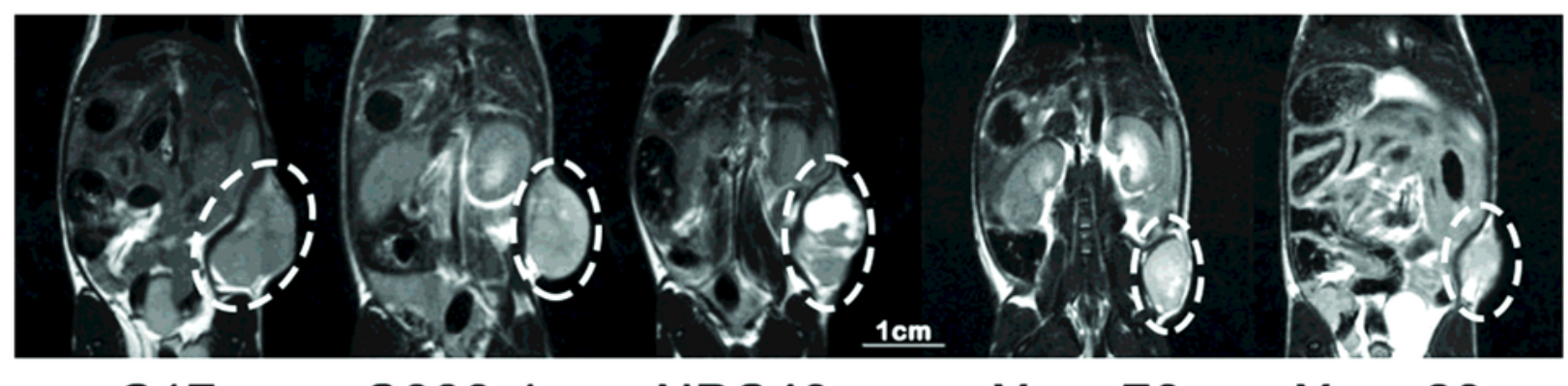

C17 C666-1 NPC43 Xeno76 Xeno23

Figure 1

Tumour growth pattern and cell doubling time of NPC xenograft models a Tumour growth of five NPC xenograft models over the period. Tumour volume was measured by MRI weekly once tumours were palpable. Observation period was selected based on a comparable size across all the models. Week2 to week5 was selected for C17, C666-1, NPC43, Xeno76 and week11 to week14 was selected for Xeno23. Tumours derived from cell lines (C17, C666-1 and NPC43) were found to have a faster growth rate than 
tumours derived from tumour blocks (Xeno76 and Xeno23) ( $\mathrm{n}=4$ for $\mathrm{C} 17 ; \mathrm{n}=5$ for other four models). $\mathrm{b}$ Representative coronal T2-weighted images of NOD.CB17-Prkdcscid/J mouse whole body (tumours indicated by white circles)

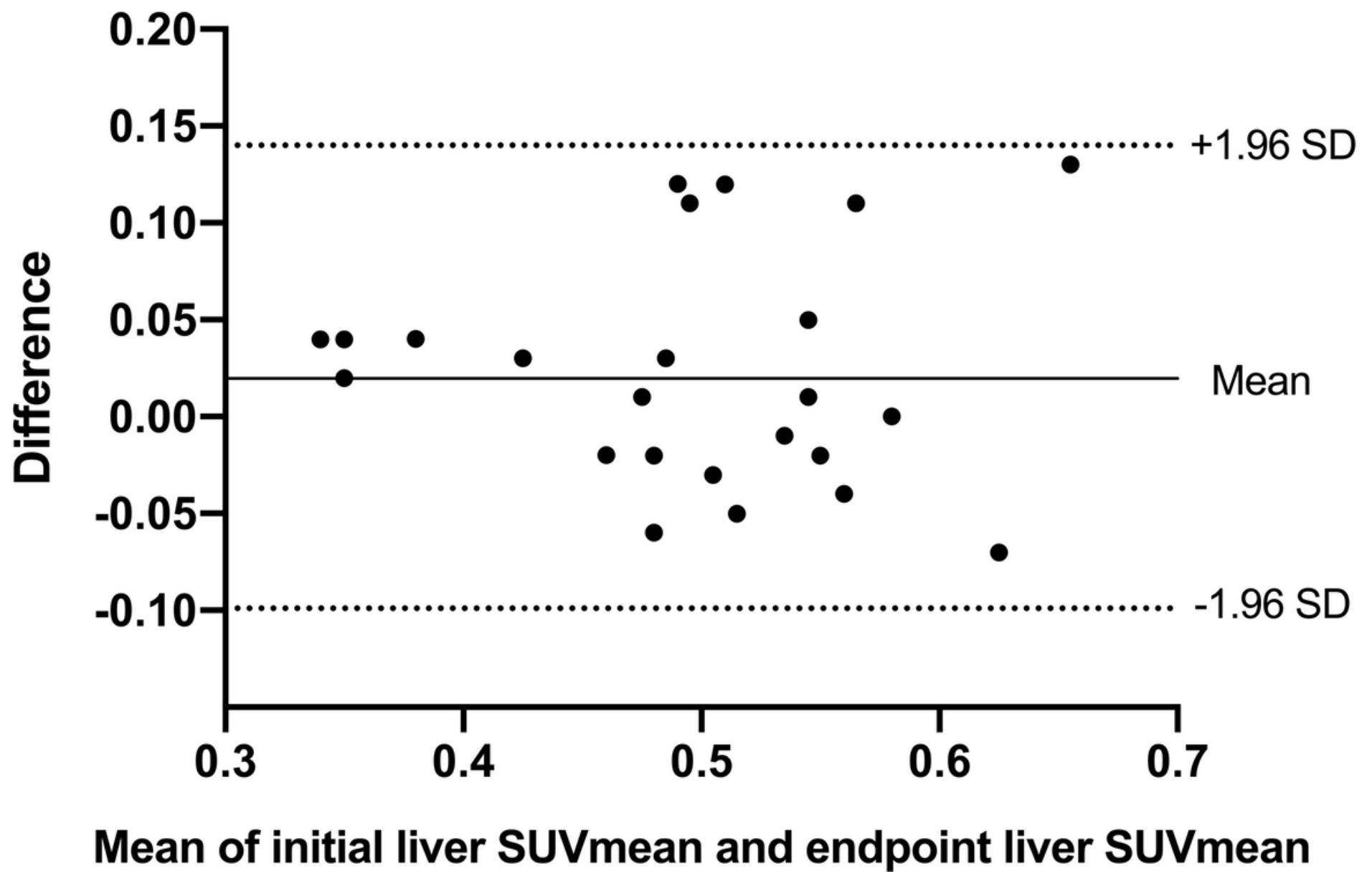

Figure 2

Repeatability of [18F]FDG microPET/MR imaging system for SUV measurement Bland-Altman plot shows the difference versus mean of initial liver [18F]FDG uptake and endpoint liver uptake. No significant difference was found between the initial and endpoint liver uptake. Each data point represents one animal 
a

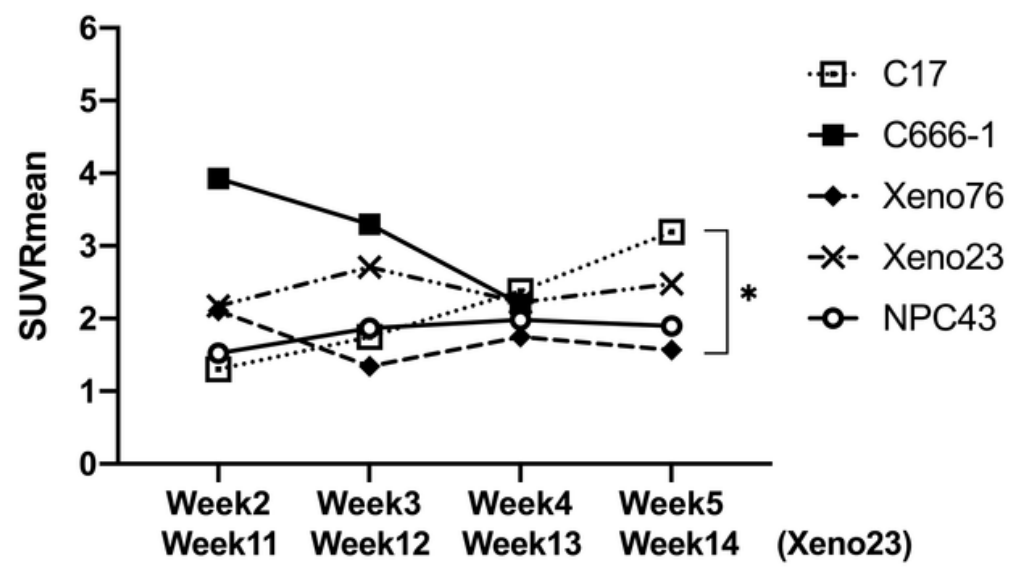

C

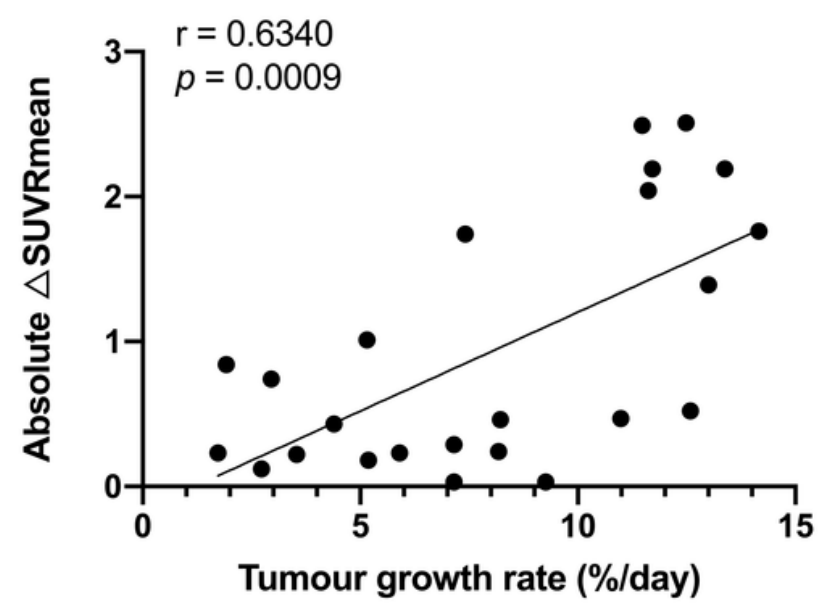

b

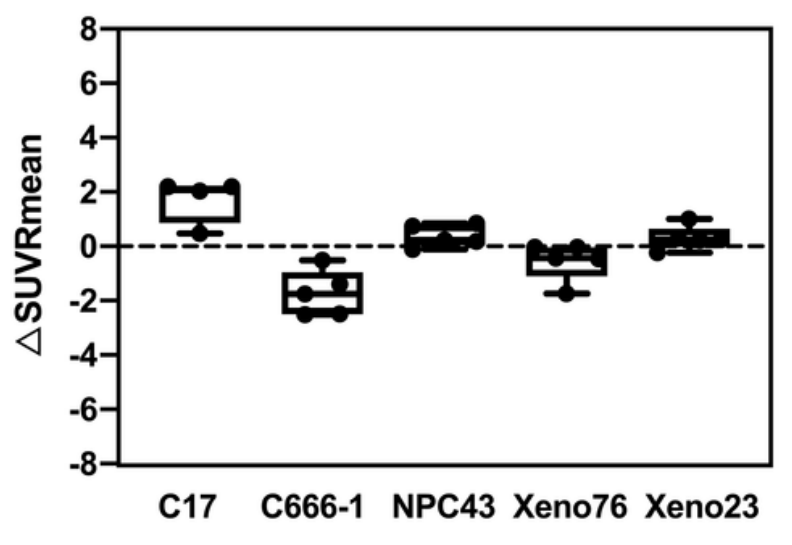

d

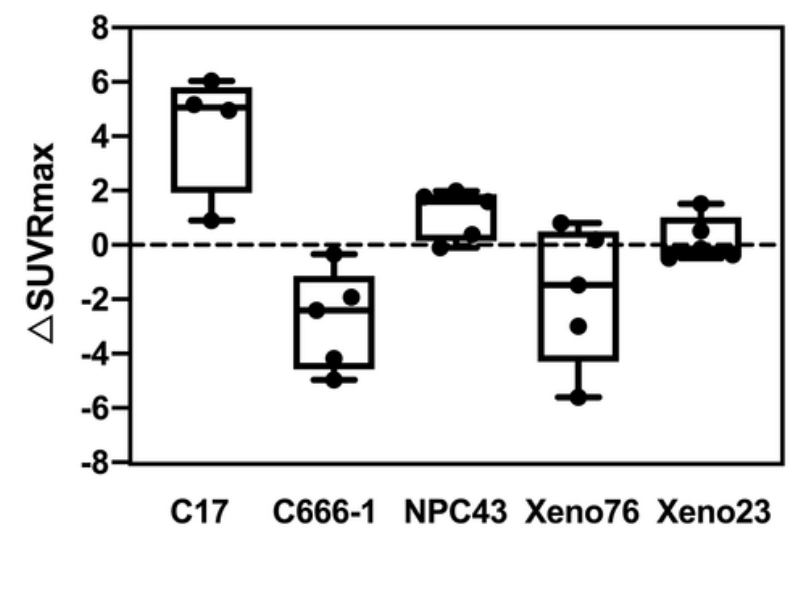

Figure 3

Longitudinal metabolic patterns of NPC xenograft models using microPET/MR a Longitudinal metabolic patterns of NPC xenograft models. Significant difference was found in SUVRmean between C17 and Xeno76 at the end of 4-week period $(p=0.0476)$. b Boxplots show the change of SUVRmean within the 4week observation period. Y-axis shows the differences of SUVR between the end and initial of the 4-week observation. c Scatter plot shows the correlation between tumour growth and absolute value of change of SUVRmean within the 4-week observation period. Data points include all the five NPC xenograft models. A significant positive correlation was found between tumour growth rate and absolute value of change of SUVRmean using Pearson's correlation. d Boxplots show the change of SUVRmax within the 4-week observation period. SUVRmean was found to be more stable than SUVRmax due to the nature of measurement. Each data point represents one animal $\left(p \otimes 0.05^{\star}, p \otimes 0.001 * * *\right)$ 

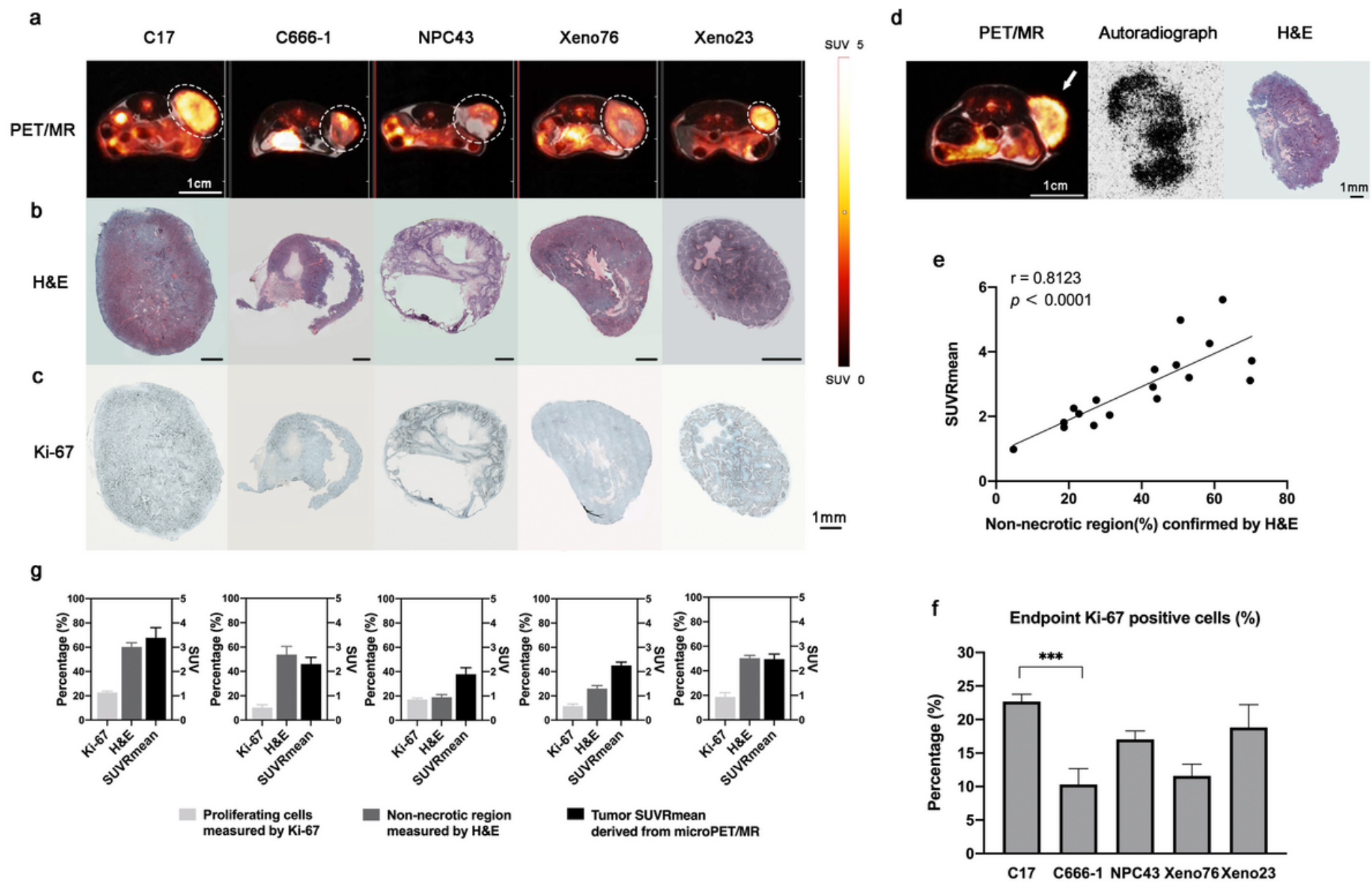

\section{Figure 4}

Tumour necrosis in NPC xenograft models was confirmed by H\&E, Ki-67 staining and autoradiograph a Representative [18F]FDG axial PET/MR images of mouse whole body (tumours indicated by white circles). b-c Representative H\&E and Ki-67 staining images for corresponding NPC xenograft models. The legend indicated by a black segment on each staining image was $1 \mathrm{~mm}$. $\mathrm{d}$ Low uptake region shown on PET/MR image is confirmed as necrosis by H\&E. Left: Representative [18F]FDG axial PET/MR image of mouse bearing with C666-1 (tumour indicated by white arrow). Middle: Corresponding ex vivo distribution of [18F]FDG. Right: Corresponding H\&E staining image. e Scatter plot shows significant positive correlation between non-necrotic region\% and SUVRmean using Pearson's correlation. Each data point represents one animal. $\mathrm{f}$ Boxplot shows the quantification of Ki-67 staining and present as the percentage of positive cells on NPC tissue slides. $\mathrm{C} 17$ has a higher number of proliferating cells than other NPC models ( $n \geq 5$ for each model, $p=0.0007$ ). $g$ Bar chart show the comparison of tumour histological characteristics and tumor SUVRmean across different NPC xenograft models $\left(p \otimes 0.001^{\star \star \star}, p \otimes\right.$ $\left.0.0001^{\star \star \star *}\right)$ 
a

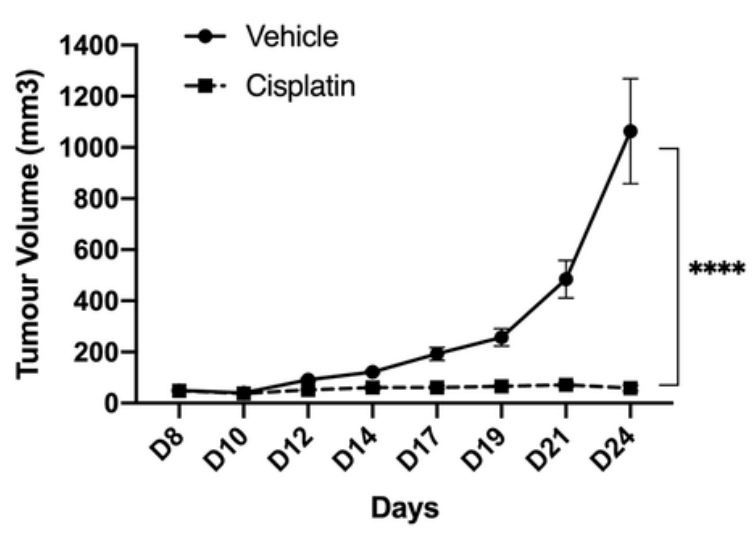

c

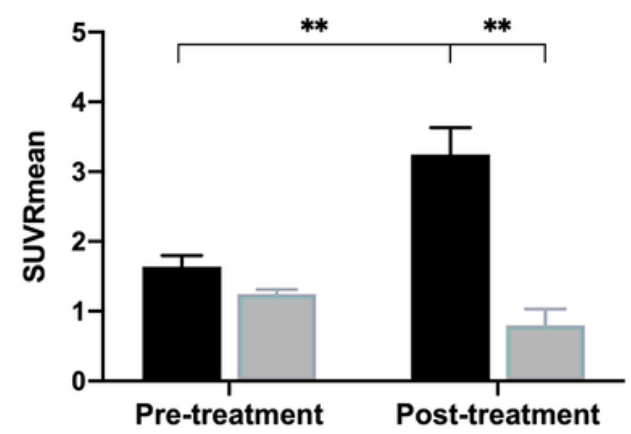

d

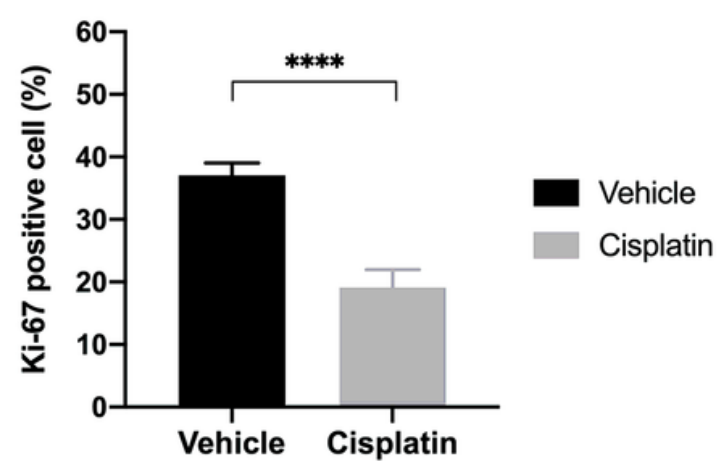

b

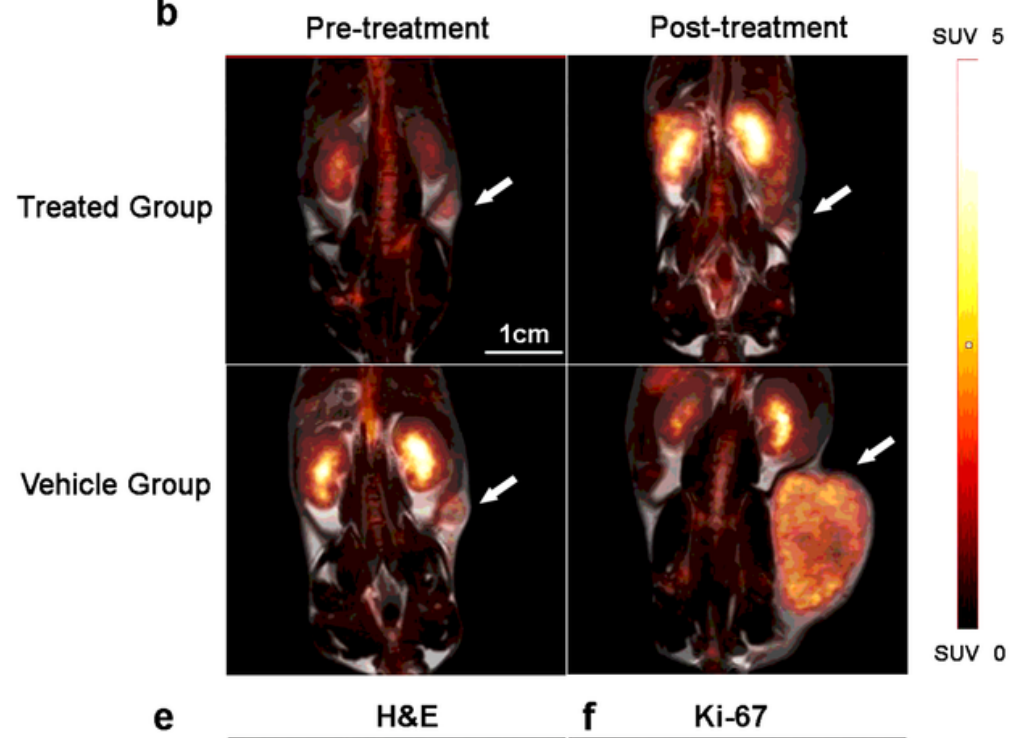

Vehicle Group

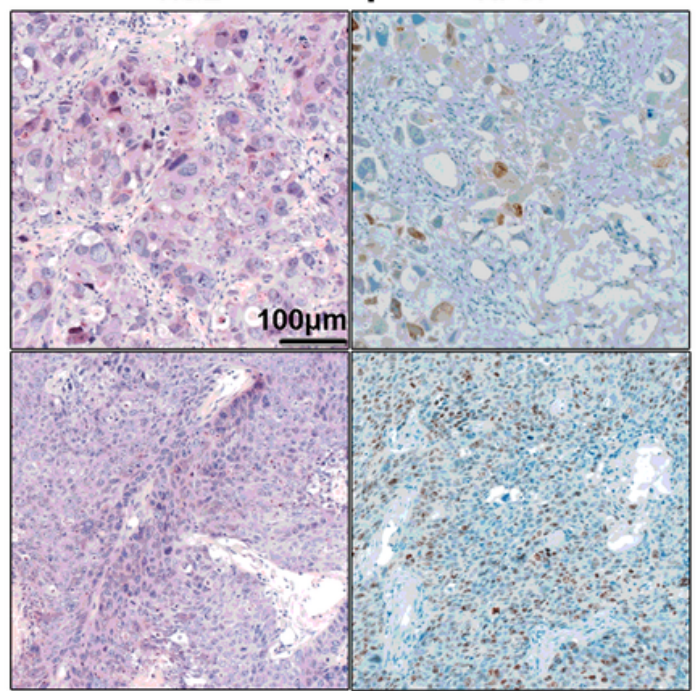

\section{Figure 5}

Cisplatin treatment on NPC xenograft model C17 a Plot shows the in vivo inhibition of growth of C17 tumour by cisplatin. A significant decrease in tumour volume was observed after weekly treatment of cisplatin for continuously 4 weeks ( $n=4$ mice per group). b Representative [18F]FDG coronal PET/MR images of mouse bearing with $\mathrm{C} 17$ (tumours indicated by white arrows) show the in vivo change of tumour volume and tumour metabolism after cisplatin treatment. c Bar chart shows the change of SUVmean before and after treatment. Statistical analysis reports a significant decrease in SUVmean after treatment compared with vehicle group $(p=0.0016)$. A significant increase was observed in SUVmean in the vehicle group $(p=0.004)$. $d$ Bar chart shows the change of cell proliferation marker Ki- 67 before and after treatment. Cisplatin treated group shows a significant decrease in Ki-67 positive cells. e-f Representative images of H\&E and Ki-67 staining of the tumour tissue from treated group and vehicle 
group. A decrease of proliferating cells was observed in cisplatin treated group compared with vehicle group $\left(p \otimes 0.01^{\star \star}, p \otimes 0.0001^{\star \star \star \star}\right)$

\section{Supplementary Files}

This is a list of supplementary files associated with this preprint. Click to download.

- Supplementarymaterials.pdf 